\title{
Expression of podoplanin and vimentin is correlated with prognosis in esophageal squamous cell carcinoma
}

\author{
MAKIKO TANAKA ${ }^{1}$, HIROSHI KIJIMA ${ }^{2}$, HIDEO SHIMADA ${ }^{3}$, \\ HIROYASU MAKUUCHI ${ }^{3}$, SOJI OZAWA $^{3}$ and SADAKI INOKUCHI $^{1}$ \\ ${ }^{1}$ Department of Critical Care and Emergency Medicine, Tokai University School of Medicine, Isehara, Kanagawa 259-1193; \\ ${ }^{2}$ Department of Pathology and Bioscience, Hirosaki University Graduate School of Medicine, Hirosaki, Aomori 036-8562; \\ ${ }^{3}$ Department of Gastroenterological Surgery, Tokai University School of Medicine, Isehara, Kanagawa 259-1193, Japan
}

Received January 4, 2014; Accepted November 12, 2014

DOI: $10.3892 / \mathrm{mmr} .2015 .3966$

\begin{abstract}
Podoplanin is a small membrane mucin, which is involved in cell migration and cancer cell invasion. However, the roles of podoplanin in esophageal squamous cell carcinoma (ESCC) are poorly understood. In the present study, 139 cases of surgically resected ESCC were analyzed and the clinicopathological significance of podoplanin membrane expression in ESCC was demonstrated. Podoplanin expression was positive in $66.2 \%$ (92/139) of ESCC samples; with weak expression in $32.4 \%$ (45/139), and strong expression in $33.8 \%$ (47/139). Membrane expression of podoplanin was significantly associated with tumor status $(\mathrm{P}=0.001)$, venous invasion $(\mathrm{P}=0.035)$ and Union for International Cancer Control stage $(\mathrm{P}=0.029)$. Patients who exhibited strong podoplanin expression, were shown to have a poorer prognosis [hazard ratio (HR), 3.949; 95\% confidence interval (CI), 2.001-7.794]. Expression of vimentin, a mesenchymal marker, was detected in 49 cases $(35.3 \%)$ and was associated with venous invasion $(\mathrm{P}=0.020)$. Vimentin-positive cases were also more likely to have a worse prognosis than vimentin-negative cases (HR, 2.161; 95\% CI, 1.300-3.592). Podoplanin membrane expression was significantly correlated with vimentin cytoplasmic expression in ESCC $(\mathrm{P}<0.001)$. The present study confirmed that podoplanin and vimentin are independent predictors of mortality (HR, 3.084; 95\% CI, 1.543-6.164). These results suggest that podoplanin membrane expression results in epithelial-mesenchymal transition and promotes aggressive invasion in human ESCC.
\end{abstract}

\footnotetext{
Correspondence to: Dr Sadaki Inokuchi, Department of Critical Care and Emergency Medicine, Tokai University School of Medicine, 143 Shimokasuya, Isehara, Kanagawa 259-1193, Japan E-mail: inokuchi@is.icc.u-tokai.ac.jp
}

Key words: podoplanin, vimentin, prognosis, esophagus, squamous cell carcinoma

\section{Introduction}

Esophageal squamous cell carcinoma (ESCC) is a common malignant tumor of the digestive tract and is associated with a poor prognosis $(1,2)$. Patients with ESCC often have a poor outcome as it is a difficult disease to diagnose at an early stage $(3,4)$. Advanced esophageal cancer carries a particularly unfavorable prognosis due to the rapid spread of the tumor beyond the esophageal wall $(5,6)$. A treatment strategy for early ESCC has been established based around endoscopic procedures, and standard curative surgery is now performed in advanced ESCC. In Japan, the total 5-year survival rate of patients with ESCC is $~ 50 \%$; that of patients with early ESCC is $>70 \%$, while in advanced cases it is $\sim 20 \%$, due to the high incidence of recurrence, metastasis and invasion of adjacent organs $(3,7)$. The prognosis of ESCC remains poor despite recent therapeutic advances. Therefore, further clinicopathological studies are required in order to analyze the mechanisms underlying local invasion and metastasis in ESCC.

Podoplanin, a $38 \mathrm{kDa}$ transmembrane protein, was identified as an independent platelet aggregation-inducing factor, and is known to be a specific marker for lymphatic vessels (8). A recent study reported that podoplanin promoted epithelial-mesenchymal transition (EMT) (9). It has also been shown to stimulate collective cell invasion and migration without EMT, by inducing a rearrangement of the actin cytoskeleton of MCF7 breast cancer cells (10). A number of studies have reported that podoplanin expression is correlated with lymph node metastasis, disease stage, lymphatic and vascular invasion, recurrence and a poor prognosis in ESCC (11,12). Vimentin is an important protein constituent of cellular intermediate filaments in normal and tumor mesenchymal cells $(13,14)$. Therefore, vimentin expression is one of the primary indicators of the development of EMT in carcinomas, which suggests a tumor with an aggressive phenotype with invasive and/or metastatic potential (15). Vimentin expression in ESCC has also been shown to be an independent predictor of lymph node metastasis $(16,17)$. However, to the best of our knowledge, there have been no clinicopathological studies investigating podoplanin and vimentin expression together in ESCC. The present study 
focused on the expression of podoplanin in the membrane, and its clinicopathological significance in the progression of ESCC.

\section{Materials and methods}

Patients. In total, 139 patients with ESCC who had undergone surgical resection of their tumor at Tokai University Hospital (Kanagawa, Japan) between January 2003 and December 2005, were enrolled into the study. The study was approved by the ethics committee of the Institutional Review Board of Tokai University Hospital (IRB no. 13R-33; Isehara, Japan). All participants provided informed consent, according to the Institutional Review Board of Tokai University Hospital. The 139 patients (128 males and 11 females; age range, $42-82$ years; mean age, 63.4 years) with ESCC underwent surgery with three-field lymph node dissection and were not treated with radiotherapy or chemotherapy prior to surgery.

Histopathological examination. Esophageal cancer specimens were routinely fixed with $10 \%$ formalin for $24-48 \mathrm{~h}$, embedded in paraffin, cut into $4-\mu \mathrm{m}$ sections and stained with hematoxylin and eosin. The tumor stage was defined according to the tumor-node-metastasis classification of the Union for International Cancer Control (UICC) (18). In addition, histological factors were graded according to the Guidelines for Clinical and Pathological Studies on Carcinoma of the Esophagus (e.g. ly, v and INF). The degree of lymph node metastasis was classified as: $\mathrm{n}(-)$, metastasis negative; or $\mathrm{n}(+)$, metastasis positive. The degree of lymphatic invasion was classified as: ly0, no lymphatic invasion; ly1, mild lymphatic invasion; ly2, moderate lymphatic invasion; or ly3, severe lymphatic invasion. The degree of venous invasion was classified as: $\mathrm{v}(-)$, invasion negative; or $\mathrm{v}(+)$, invasion positive. Tumor infiltrative patterns (INF) at the invasive front were classified into three groups according to the general criteria described by the Japanese Gastric Cancer Association (19): INFa, cancer nests demonstrate expanding growth and have a clear border with pre-vesicular adipose tissue; INFb, cancer nests are intermediate between those of INFa and INFc; and INFc, scirrhous growth, in which cancer nests exhibit invasive growth and where the border with adipose tissue is unclear.

Immunohistochemical analysis. An representative specimen for immunohistochemical analysis was selected from each patient with ESCC. Sections $(5 \mu \mathrm{m})$ were mounted on silane-coated glass slides, deparaffinized and autoclaved (ES-215, high-pressure steam sterilizer, TOMY, Tokyo, Japan) at $121^{\circ} \mathrm{C}$ for $10 \mathrm{~min}$ for antigen retrieval. The primary antibodies used in the immunohistochemical analyses were mouse monoclonal anti-podoplanin (clone D2-40; 3X dilution; cat. no. 413451; Nichirei Pharmaceutical, Tokyo, Japan) and mouse monoclonal anti-vimentin (clone v9; X100 dilution; cat. no. MO725; Dako Denmark A/S, Glostrup, Denmark). Immunoreactivity was detected using the avidin-biotin method (Vectastain Elite ABC kit, Vector Laboratories, Inc., Burlingame, CA, USA). Immunohistochemical images were captured using a digital microscope camera system (BX50 microscope and DP70 digital camera; Olympus Corporation, Tokyo, Japan).
Evaluation of immunohistochemistry. For the analysis of the immunohistochemical expression of podoplanin, staining intensity was determined at the invasive front of ESCC (Fig. 1), and was classified into the following three criteria: 1) negative, incomplete membrane expression of $<10 \%$ cancer cells at the invasive front ( Fig. 1D); 2) weak, complete membrane expression of $<10 \%$ cancer cells (Fig. 1E); 3) strong, complete membrane expression of $\geq 10 \%$ cancer cells (Fig. 1F). Vimentin expression was determined by the presence of cytoplasmic staining, in particular in the tumor/stoma interface at the invasive front of the cancer cells, and divided into the following two criteria: Negative, $<10 \%$ vimentin expression at the invasive front of the cancer cells and positive, $\geq 10 \%$ vimentin expression at the invasive front (Fig. 1E).

Statistical analysis. Univariate analysis was performed using the $\chi^{2}$ test. Cox proportional hazard regression analysis was used to determine the net effects of each predictor while controlling the effects of the other factors by uni- and multivariate analysis. Independent prognostic factors were analyzed by the Cox proportional hazard regression model. Hazard ratios (HRs) and associated 95\% confidence intervals (CIs) were used to assess the independent contributions of significant factors. $\mathrm{P}<0.05$ was considered to indicate a statistically significant difference. Overall survival was measured from the date of surgery to mortality from all causes. Survival curves were calculated using Kaplan-Meier methods and analyzed using the log-rank test. All analyses were performed using SPSS statistical software, version 21 (IBM SPSS, Armonk, NY, USA).

\section{Results}

Histological expression of podoplanin and vimentin. Podoplanin was strongly expressed in the endothelial cells of lymphatic vessels, which were examined as internal controls. Non-neoplastic mucosa, obtained from the surgically resected esophagus with ESCC, exhibited weak podoplanin expression at the basal layer adjacent to the connective tissue papillae. Podoplanin expression was positive in $66.2 \%(92 / 139)$ of samples from patients with ESCC (Fig. 2); weak expression was observed in $32.4 \%(45 / 139)$ and strong expression in $33.8 \%$ (47/139; Fig. 1). Vimentin expression was observed in the esophageal lamina propria, but was not detected in non-neoplastic esophageal epithelium (Fig. 2). Vimentin expression at the cancer invasive front was detected in $35.3 \%$ (49/139) of samples from patients with ESCC.

\section{Analysis of clinicopathological findings from ESCC using} $\chi^{2}$ statistics. The associations between podoplanin and clinicopathological features, are summarized in Table I. Strong podoplanin expression was significantly correlated with tumor status $(\mathrm{P}=0.001)$, venous invasion $(\mathrm{P}=0.035)$ and UICC stage $(\mathrm{P}=0.029)$. The associations between Vimentin and clinicopathological features are summarized in Table II. Vimentin expression was significantly associated with venous invasion $(\mathrm{P}=0.020)$. The association between podoplanin and vimentin is summarized in Table III. Podoplanin membrane expression was strongly correlated with vimentin 

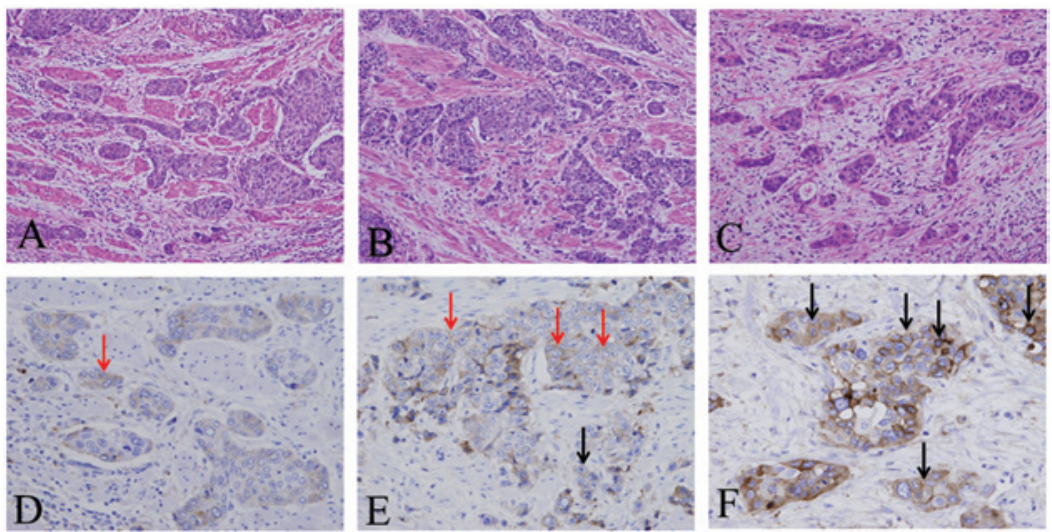

Figure 1. Immunohistochemical expression of podoplanin. Squamous cell carcinoma cells invasion of the stroma. Three representative cases, (A), (B) and (C). Hematoxylin and eosin staining (magnification, x20). (D) Section from same sample as image (A). Negative, podoplanin is expression in incomplete membrane at ESCC. (E) Section from same sample as image (B). Weak, podoplanin is expression in incomplete membrane and/or complete membrane at ESCC. (F) Section from same sample as image (C). Strong, podoplanin is expression complete membrane at ESCC. Red arrows indicate expression in incomplete cell membrane, and black arrows are expression at complete cell membrane (magnification, x40). ESCC, esophageal squamous cell carcinoma.
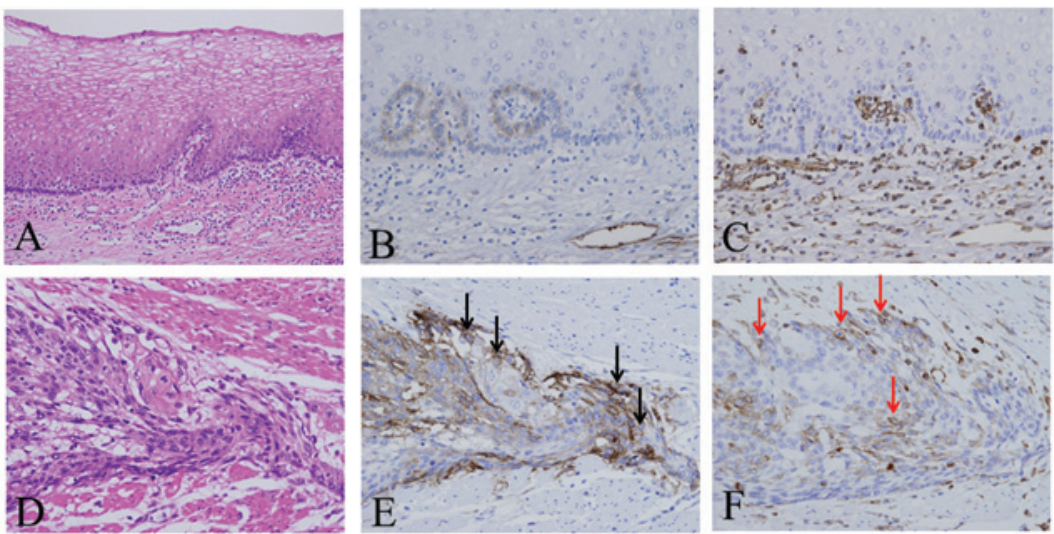

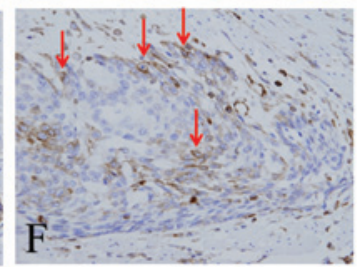

Figure 2. Immunohistochemical expression of podoplanin and vimentin. (A) Non-neoplastic esophageal mucosa consists of epithelium and lamina propria (H\&E staining). (B) Podoplanin is strongly expressed in endothelial cells of lymphatic vessels but weakly expressed in the epithelial basal layers. (C) Vimentin staining was observed in the lamina propria, but was not detected in the epithelium. (D) Esophageal squamous cell carcinoma invasion of the stroma (H\&E staining). (E) Podoplanin is strongly expressed at the invasive front of the carcinoma (black arrows). (F) Vimentin is expressed in a proportion of the carcinoma cells (red arrows), as well as in the stromal tissue (magnification, $\mathrm{x} 40$ ). H\&E, hematoxylin and eosin.

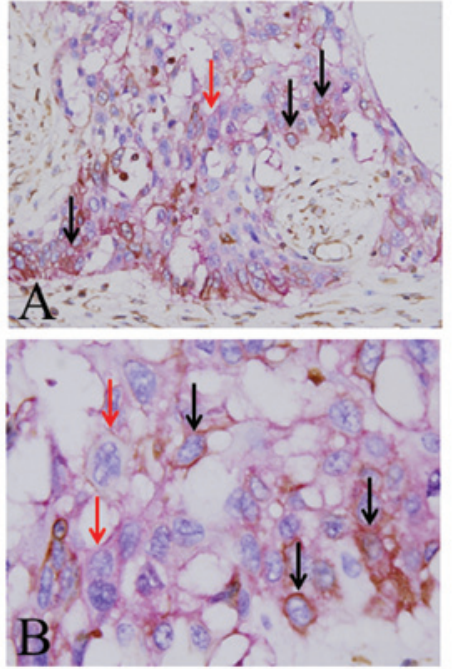

Figure 3. Double immunohistochemical staining of podoplanin (red phosphatase staining) and vimentin (brown peroxidase staining). A number of cancer cells expressed podoplanin and vimentin (black arrows). Podoplanin expression is observed at the cell membranes of carcinoma cells (red arrows). (A) Low power magnification (x40) and (B) high power magnification (x100).
$\mathbf{A}$

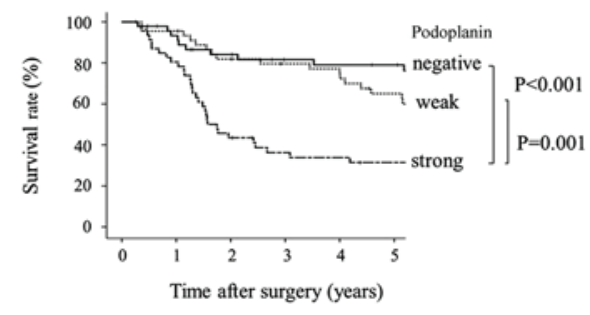

B

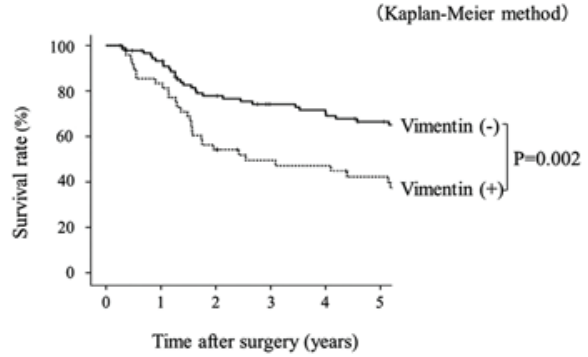

Figure 4. Kaplan-Meier survival curves. (A) Association between podoplanin expression and overall survival in ESCC. (B) Association between vimentin expression and survival in ESCC. ESCC, esophageal squamous cell carcinoma. 
Table I. Podoplanin membrane expression and clinicopathological factors of esophageal squamous cell carcinoma.

\begin{tabular}{|c|c|c|c|c|c|}
\hline \multirow[b]{2}{*}{ Variable } & \multirow[b]{2}{*}{ Number of patients } & \multicolumn{3}{|c|}{ Podoplanin staining intensity } & \multirow[b]{2}{*}{ P-value } \\
\hline & & Negative & Weak & Strong & \\
\hline Age at surgery (years) & & & & & 0.406 \\
\hline$<63$ & $66(47.5)$ & $21(31.8)$ & $19(28.8)$ & $26(39.4)$ & \\
\hline$\geq 63$ & $73(52.5)$ & $26(35.6)$ & $26(35.6)$ & $21(28.8)$ & \\
\hline Gender & & & & & 0.696 \\
\hline Male & $128(92.1)$ & $42(32.8)$ & $42(32.8)$ & $44(34.4)$ & \\
\hline Female & $11(7.9)$ & $5(45.4)$ & $3(27.3)$ & $3(27.3)$ & \\
\hline Tumor status & & & & & 0.001 \\
\hline $\mathrm{T} 1$ & $48(34.5)$ & $24(50.0)$ & $17(35.4)$ & $7(14.6)$ & \\
\hline $\mathrm{T} 2$ & $25(18.0)$ & $4(16.0)$ & $13(52.0)$ & $8(32.0)$ & \\
\hline $\mathrm{T} 3$ & $61(43.9)$ & $19(31.1)$ & $14(23.0)$ & $28(45.9)$ & \\
\hline $\mathrm{T} 4$ & $5(3.6)$ & $0(0.0)$ & $1(20.0)$ & $4(80.0)$ & \\
\hline Lymph node metastasis & & & & & 0.164 \\
\hline $\mathrm{n}(-)$ & $43(30.9)$ & $18(40.9)$ & $16(36.4)$ & $10(22.7)$ & \\
\hline $\mathrm{n}(+)$ & $96(69.1)$ & $29(30.5)$ & $29(30.5)$ & $37(39.0)$ & \\
\hline Lymphatic invasion & & & & & 0.870 \\
\hline ly $(0,1)$ & $87(62.6)$ & $30(34.5)$ & $29(33.3)$ & $28(32.2)$ & \\
\hline ly $(2,3)$ & $52(37.4)$ & $17(32.7)$ & $16(30.8)$ & $19(36.5)$ & \\
\hline Venous invasion & & & & & 0.035 \\
\hline $\mathrm{v}(-)$ & $52(37.4)$ & $23(44.2)$ & $18(34.6)$ & $11(21.2)$ & \\
\hline $\mathrm{v}(+)$ & $87(62.6)$ & $24(27.6)$ & $27(31.0)$ & $36(41.4)$ & \\
\hline Histological differentiation & & & & & 0.159 \\
\hline Well & $38(27.3)$ & $9(23.7)$ & $13(34.2)$ & $16(42.1)$ & \\
\hline Mod & $77(55.4)$ & $25(32.4)$ & $26(33.8)$ & $26(33.8)$ & \\
\hline Poor & $24(17.3)$ & $13(54.2)$ & $6(25.0)$ & $5(20.8)$ & \\
\hline Infiltration pattern & & & & & 0.398 \\
\hline $\inf a, b$ & $97(69.8)$ & $36(37.1)$ & $31(32.0)$ & $30(30.9)$ & \\
\hline $\inf c$ & $42(30.2)$ & $11(26.2)$ & $14(33.3)$ & $17(40.5)$ & \\
\hline UICC stage & & & & & 0.029 \\
\hline IA, IB & $37(26.6)$ & $16(43.2)$ & $14(37.9)$ & $7(18.9)$ & \\
\hline IIA, IIB & $36(25.9)$ & $10(27.8)$ & $16(44.4)$ & $10(27.8)$ & \\
\hline IIIA, IIIB, IIIC & $66(47.5)$ & $21(31.8)$ & $15(22.7)$ & $30(45.5)$ & \\
\hline
\end{tabular}

Numbers in brackets represent the percentages of patients/samples in that category. $n$, lymph node metastasis; ly, lymphatic invasion; $v$, venous invasion; UICC, Union for International Cancer Control.

cytoplasmic expression in samples from patients with ESCC $(\mathrm{P}<0.001)$. Immunohistochemical double-staining demonstrated co-expression of podoplanin and vimentin in ESCC (Fig. 3).

Correlation between prognosis and expression of podoplanin and vimentin in ESCC. Patients exhibiting strong podoplanin expression had significantly poorer overall survival rates than those with negative or weak expression of podoplanin $(<0.001, \mathrm{P}=0.001$, log-rank test, Fig. $4 \mathrm{~A})$. Patients from whom ESCC samples were vimentin-positive also had lower overall survival rates than patients who were negative for vimentin expression ( $\mathrm{P}=0.002$, log-rank test, Fig. 4B). Multivariate analysis demonstrated that strong podoplanin expression (HR, 3.084; 95\% CI, 1.543-6.164) and lymph node metastasis (HR, 6.132; 95\% CI, 2.355-15.916) were independent predictors of mortality in ESCC (Table IV). In addition, multivariate analysis showed that vimentin expression (HR, 
Table II. Vimentin expression and clinicopathological factors of esophageal squamous cell carcinoma.

\begin{tabular}{|c|c|c|c|c|}
\hline Variable & Number of patients & Vimentin (-) & Vimentin (+) & P-value \\
\hline Age at surgery (years) & & & & 0.794 \\
\hline$<63$ & $66(47.5)$ & $42(63.6)$ & $24(36.4)$ & \\
\hline$\geq 63$ & $73(52.5)$ & $48(65.8)$ & $25(34.2)$ & \\
\hline Gender & & & & 0.163 \\
\hline Male & $128(92.1)$ & $85(66.4)$ & $43(33.6)$ & \\
\hline Female & $11(7.9)$ & $5(45.5)$ & $6(54.5)$ & \\
\hline Tumor status & & & & 0.143 \\
\hline $\mathrm{T} 1$ & $48(34.5)$ & $37(77.1)$ & $11(22.9)$ & \\
\hline $\mathrm{T} 2$ & $25(18.0)$ & $16(64.0)$ & $9(36.0)$ & \\
\hline $\mathrm{T} 3$ & $61(43.9)$ & $34(55.7)$ & $27(44.3)$ & \\
\hline $\mathrm{T} 4$ & $5(3.6)$ & $3(60.0)$ & $2(40.0)$ & \\
\hline Lymph node metastasis & & & & 0.180 \\
\hline n (-) & $44(31.7)$ & $32(72.7)$ & $12(27.3)$ & \\
\hline $\mathrm{n}(+)$ & $95(68.3)$ & $58(61.1)$ & $37(38.9)$ & \\
\hline Lymphatic invasion & & & & 0.178 \\
\hline ly $(0,1)$ & $87(62.6)$ & $60(69.0)$ & $27(31.0)$ & \\
\hline ly $(2,3)$ & $52(37.4)$ & $30(57.7)$ & $22(42.3)$ & \\
\hline Venous invasion & & & & 0.020 \\
\hline $\mathrm{V}(-)$ & $52(37.4)$ & $40(76.9)$ & $12(23.1)$ & \\
\hline $\mathrm{v}(+)$ & $87(62.6)$ & $50(57.5)$ & $37(42.5)$ & \\
\hline Histological differentiation & & & & 0.582 \\
\hline Well & $38(27.3)$ & $22(57.9)$ & $16(42.1)$ & \\
\hline Mod & $77(55.4)$ & $52(67.5)$ & $25(32.5)$ & \\
\hline Poor & $24(17.3)$ & $16(66.7)$ & $8(33.3)$ & \\
\hline Infiltration pattern & & & & 0.105 \\
\hline $\inf a, b$ & $97(69.8)$ & $67(69.1)$ & $30(30.9)$ & \\
\hline $\inf c$ & $42(30.2)$ & $23(54.8)$ & $19(45.2)$ & \\
\hline UICC stage & & & & 0.231 \\
\hline IA, IB & $37(26.6)$ & $27(73.0)$ & $10(27.0)$ & \\
\hline IIA, IIB & $36(25.9)$ & $25(69.4)$ & $11(30.6)$ & \\
\hline IIIA, IIIB, IIIC & $66(47.5)$ & $38(57.6)$ & $28(42.4)$ & \\
\hline
\end{tabular}

Numbers in brackets represent the percentages of patients/samples in that category. $\mathrm{n}$, lymph node metastasis; ly, lymphatic invasion; v, venous invasion; UICC, Union for International Cancer Control.

Table III. Podoplanin membrane expression and vimentin expression in esophageal squamous carcinoma.

\begin{tabular}{|c|c|c|c|c|c|}
\hline \multirow[b]{2}{*}{ Variable } & \multirow[b]{2}{*}{ Number of patients } & \multicolumn{3}{|c|}{ Podoplanin staining intensity } & \multirow[b]{2}{*}{ P-value } \\
\hline & & Negative & Weak & Strong & \\
\hline Vimentin & & & & & $<0.001$ \\
\hline Negative & $90(64.7)$ & $42(46.7)$ & $31(34.4)$ & $17(18.9)$ & \\
\hline Positive & $49(35.3)$ & $5(10.2)$ & $14(28.6)$ & $30(61.2)$ & \\
\hline
\end{tabular}

Numbers in brackets represent the percentages of patients/samples in that category. 
Table IV. Multivariate analysis of clinicopathological factors and patients' survival of esophageal squamous cell carcinoma.

\begin{tabular}{|c|c|c|c|c|}
\hline Variable & Number of patients & Hazard ratio & $95 \%$ confidence interval & P-value \\
\hline \multicolumn{5}{|c|}{ Podoplanin staining intensity } \\
\hline Negative (reference) & $47(33.8)$ & & & \\
\hline Weak & $45(32.4)$ & 1.509 & $0.717-3.174$ & 0.278 \\
\hline Strong & $47(33.8)$ & 3.084 & $1.543-6.164$ & 0.001 \\
\hline Age at surgery (years) & & & & 0.551 \\
\hline$<63$ & $66(47.5)$ & 0.856 & $0.514-1.426$ & \\
\hline$\geq 63$ & $73(52.5)$ & & & \\
\hline Gender & & & & 0.604 \\
\hline Male & $128(92.1)$ & 0.685 & $0.164-2.868$ & \\
\hline Female & $11(7.9)$ & & & \\
\hline Tumor status & & & & 0.123 \\
\hline $\mathrm{T} 1, \mathrm{~T} 2$ & $73(52.5)$ & 1.587 & $0.882-2.854$ & \\
\hline $\mathrm{T} 3, \mathrm{~T} 4$ & $66(47.5)$ & & & \\
\hline Lymph node metastasis & & & & $<0.001$ \\
\hline n (-) & $44(31.7)$ & 6.123 & $2.355-15.916$ & \\
\hline $\mathrm{n}(+)$ & $95(68.3)$ & & & \\
\hline
\end{tabular}

Numbers in brackets represent the percentages of patients/samples in that category. $\mathrm{n}$, lymph node metastasis.

Table V. Multivariate analysis of clinicopathological factors and patients' survival of esophageal squamous cell carcinoma.

\begin{tabular}{|c|c|c|c|c|}
\hline Variable & Number of patients & Hazard ratio & $95 \%$ confidence interval & P-value \\
\hline Vimentin & & & & 0.009 \\
\hline Negative & $90(64.7)$ & 2.008 & $1.191-3.384$ & \\
\hline Positive & $49(35.3)$ & & & \\
\hline Age at surgery (years) & & & & 0.528 \\
\hline$<63$ & $66(47.5)$ & 0.849 & $0.510-1.412$ & \\
\hline$\geq 63$ & $73(52.5)$ & & & \\
\hline Gender & & & & 0.266 \\
\hline Male & $128(92.1)$ & 0.441 & $0.104-1.865$ & \\
\hline Female & $11(7.9)$ & & & \\
\hline Tumor status & & & & 0.033 \\
\hline $\mathrm{T} 1, \mathrm{~T} 2$ & $73(52.5)$ & 1.830 & $1.049-3.194$ & \\
\hline $\mathrm{T} 3, \mathrm{~T} 4$ & $66(47.5)$ & & & \\
\hline Lymph node metastasis & & & & $<0.001$ \\
\hline n (-) & $90(64.7)$ & 5.777 & $2.221-15.028$ & \\
\hline $\mathrm{n}(+)$ & $49(35.3)$ & & & \\
\hline
\end{tabular}

Numbers in brackets represent the percentages of patients/samples in that category. $\mathrm{n}$, lymph node metastasis.

2.008; 95\% CI, 1.191-3.384), tumor status (HR, 1.830; 95\% CI, 1.049-3.194) and lymph node metastasis (HR, 5.77; 95\% CI, 2.221-15.028) were independent predictors of mortality in ESCC (Table V).

\section{Discussion}

Recently, podoplanin has been shown to be a candidate marker for cancer stem cells, which is associated with 
cancer cell invasion and migration, as well as prognosis, in a number of types of cancer, including esophageal squamous cell carcinoma, lung squamous cell carcinoma and oral cancer $(11,12,20-22)$. In the present study, the focus was on the expression of podoplanin in the cell membrane at the invasive front of ESCC tumors, since the podoplanin molecule is a transmembrane protein. The expression of podoplanin on the cell membrane was positively correlated with tumor status, venous invasion and UICC stage, and was associated with a poor prognosis in ESCC. Strong podoplanin membrane expression was an independent predictor of mortality in patients with ESCC (HR 3.084, 95\% CI, 1.543-6.164). To the best of our knowledge, this is the first study to report the association between podoplanin membrane expression and the degree or presence of certain clinicopathological factors. Studies have previously described podoplanin expression in cancer cells, but have not discussed podoplanin membrane expression specifically $(11,12)$.

Rahadiani et al (11) reported that high podoplanin expression was significantly correlated with tumor status, depth of invasion, and lymphatic and vascular invasion, and was associated with a poorer prognosis in ESCC. These authors demonstrated that podoplanin was involved in cancer cell invasion and tumorigenesis through the use of experimental procedures, such as the Matrigel invasion assay and an in vivo mouse study. Tong et al (12) reported that podoplanin expression was correlated with lymph node metastasis, UICC stage and the immunoreactivity score, and was associated with a poor prognosis in ESCC.

Several studies have clarified that podoplanin expression promotes EMT at molecular/histopathological levels (9). The present study focused on membrane expression of podoplanin, as membrane expression has been suggested to have significant roles in EMT (9) and cell invasion processes (10). In cancer cells, EMT is a phenotypic change, by which epithelial cells lose their polarity and epithelial markers, such as E-cadherin, and acquire migratory factors that are characteristic of fibroblasts, such as snail and vimentin (23-27). Vimentin expression is an important indicator of EMT in carcinomas (27). This transition suggests the development of an aggressive phenotype with increased invasive and metastatic potential (17). Increased vimentin expression has been reported in a number of epithelial cancers, including breast cancer, lung cancer and esophageal squamous cell carcinoma $(16,28,29)$. In the present study, it was also demonstrated that vimentin expression is associated with venous invasion and that it is an independent predictor of prognosis in ESCC. It is hypothesized that podoplanin is likely to be important in the process of EMT, as a number of cancer cells in ESCC co-expressed podoplanin and vimentin. A proportion of podoplanin-positive cancer cells also expressed vimentin, suggesting that podoplanin may result in vimentin-associated EMT. EMT is understood to induce a more aggressively invasive and malignant phenotype in ESCC. Future studies should also analyze the expression of EMT markers, such as N-cadherin, snail and fibronectin, and the epithelial marker, E-cadherin, in ESCC. Finally, in the present study, the membrane expression of podoplanin was shown to be a novel immunohistochemical indicator of a highly malignant phenotype of human ESCC.

\section{References}

1. Chino O, Kijima H, Shimada H, et al: Accumulation of p53 in esophageal squamous cell carcinoma. Int J Mol Med 8: 359-363, 2001.

2. Oshiba G, Kijima H, Himeno S, et al: Stromal thrombospondin-1 expression is correlated with progression of esophageal squamous cell carcinomas. Anticancer Res 19: 4375-4378, 1999.

3. Makuuchi H, Shimada H, Mizutani K, et al: Clinical pathological analysis of surgically resected superficial esophageal carcinoma to determine criteria for deciding on treatment strategy. Diagn Ther Endosc 3: 211-220, 1997.

4. Shimada H, Makuuchi $\mathrm{H}$, Ozawa S, et al: Technique of the double-channel ESD method performed with an EEMR tube. Esophagus 8: 67-70, 2011.

5. Prognostic significance of CyclinD1 and E-Cadherin in patients with esophageal squamous cell carcinoma: multiinstitutional retrospective analysis. Research Committee on Malignancy of Esophageal Cancer, Japanese Society for Esophageal Diseases. J Am Coll Surg 192: 708-718, 2001.

6. Yoneda M, Fujiwara H, Furutani A, et al: Prognostic impact of tumor IL-6 expression after preoperative chemoradiotherapy in patients with advanced esophageal squamous cell carcinoma. Anticancer Res 33: 2699-2705, 2013.

7. Ito E, Ozawa S, Kijima H, et al: New invasive patterns as a prognostic factor for superficial esophageal cancer. J Gastroenterol 47 : 1279-1289, 2012

8. Kaneko MK, Kato Y, Kitano T and Osawa M: Conservation of a platelet activating domain of Aggrus/podoplanin as a platelet aggregation-inducing factor. Gene 378: 52-57, 2006.

9. Martin-Villar E, Megías D, Castel S, et al: Podoplanin binds ERM proteins to activate RhoA and promote epithelial-mesenchymal transition. J Cell Sci 119: 4541-4553, 2006.

10. Wicki A,Lehembre F, WickN, et al: Tumor invasion in the absence of epithelial-mesenchymal transition: podoplanin-mediated remodeling of the actin cytoskeleton. Cancer Cell 9: 261-272, 2006.

11. Rahadiani N, Ikeda J, Makino T, et al: Tumorigenic role of podoplanin in esophageal squamous-cell carcinoma. Ann Surg Oncol 17: 1311-1323, 2010.

12. Tong L, Yuan S, Feng F and Zhang H: Role of podoplanin expression in esophageal squamous cell carcinoma: a retrospective study. Dis Esophagus 25: 72-80, 2012.

13. Zeisberg $M$ and Neilson EG: Biomarkers for epithelial-mesenchymal transitions. J Clin Invest 119: 1429-1437, 2009.

14. Thiery JP: Epithelial-mesenchymal transitions in tumour progression. Nat Rev Cancer 2: 442-454, 2002.

15. Satelli A and Li S: Vimentin in cancer and its potential as a molecular target for cancer therapy. Cell Mol Life Sci 68: 3033-3046, 2011.

16. Jin H, Morohashi S, Sato F, et al: Vimentin expression of esophageal squamous cell carcinoma and its aggressive potential for lymph node metastasis. Biomed Res 31: 105-112, 2010.

17. Mendez MG, Kojima S and Goldman RD: Vimentin induces changes in cell shape, motility, and adhesion during the epithelial to mesenchymal transition. FASEB J 24: 1838-1851, 2010.

18. Sobin LH, Gospodrowicz MK and Wittekind C (eds): TNM Classification of Malignant Tumors. 7th edition. Wiley, Hoboken, NJ, USA, 2009

19. Japanese Gastric Cancer Association: Japan classification of gastric carcinoma, 2nd English edition. Gastric Cancer 1: 10-24, 1998.

20. Shimada Y, Ishii G, Nagai K, et al: Expression of podoplanin, CD44, and p63 in squamous cell carcinoma of the lung. Cancer Sci 100: 2054-2059, 2009.

21. Yuan P, Temam S, El-Naggar A, et al: Overexpression of podoplanin in oral cancer and its association with poor clinical outcome. Cancer 107: 563-569, 2006.

22. Suzuki H, Onimaru M, Yonemitsu Y, et al: Podoplanin in cancer cells is experimentally able to attenuate prolymphangiogenic and lymphogenous metastatic potentials of lung squamoid cancer cells. Mol Cancer 9: 287, 2010.

23. Sudo T, Iwaya T, Nishida N, et al: Expression of mesenchymal markers vimentin and fibronectin: the clinical significance in esophageal squamous cell carcinoma. Ann Surg Oncol 20 (Suppl 3): S324-S335, 2013.

24. Wicki A and Christofori G: The potential role of podoplanin in tumour invasion. Br J Cancer 96: 1-5, 2007. 
25. Myong NH: Loss of E-cadherin and acquisition of vimentin in epithelial-mesenchymal transition are noble indicators of uterine cervix cancer progression. Korean J Pathol 46: 341-348, 2012.

26. Le Bras GF, Allison GL, Richards NF, et al: CD44 upregulation in E-cadherin-negative esophageal cancers results in cel invasion. PLoS One 6: e27063, 2011.

27. Usami Y, Satake S, Nakayama F, et al: Snail-associated epithelial-mesenchymal transition promotes oesophageal squamous cell carcinoma motility and progression. J Pathol 215: 330-339, 2008
28. Hendrix MJ, Seftor EA, Seftor RE and Trevor KT: Experimental co-expression of vimentin and keratin intermediate filaments in human breast cancer cells results in phenotypic interconversion and increased invasive behavior. Am J Pathol 150: 483-495, 1997.

29. Dauphin M, Barbe C, Lemaire S, et al: Vimentin expression predicts the occurrence of metastases in non small cell lung carcinomas. Lung Cancer 81: 117-122, 2013. 